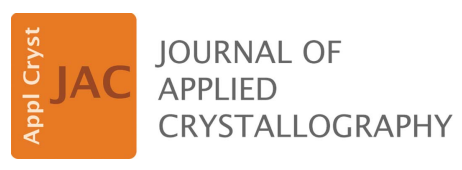

ISSN 1600-5767

Received 5 May 2018

Accepted 29 October 2018

Edited by E. P. Gilbert, ANSTO, Kirrawee DC, Australia

Keywords: small-angle neutron scattering; SANS; calibration; wavelength; absolute intensity.

\section{Calibration of the Suanni small-angle neutron scattering instrument at the China Mianyang Research Reactor}

\author{
Qiang Tian, ${ }^{\mathrm{a}, \mathrm{b} *}$ Guanyun Yan, ${ }^{\mathrm{b}}$ Liangfei Bai, ${ }^{\mathrm{b}}$ Jie Chen, ${ }^{\mathrm{b}}$ Dong Liu, ${ }^{\mathrm{b}}$ Liang Chen, \\ Liangwei Sun, ${ }^{\mathrm{b}}$ Chaoqiang Huang, ${ }^{\mathrm{b}}$ Bo Chen, ${ }^{\mathrm{b}}$ Gergely Nagy, ${ }^{\mathrm{c}, \mathrm{d}}$ András Wacha ${ }^{\mathrm{e}}$ \\ and László Almásy ${ }^{\mathrm{a}, \mathrm{c} *}$
}

\begin{abstract}
${ }^{a}$ State Key Laboratory of Environment-Friendly Energy Materials, Southwest University of Science and Technology, Mianyang 621010, People's Republic of China, '⿳亠丷厂 Key Laboratory of Neutron Physics and Institute of Nuclear Physics and Chemistry, China Academy of Engineering Physics, Mianyang 621999, People's Republic of China, 'Institute for Solid State Physics and Optics, Wigner Research Centre for Physics, PO Box 49, H-1525 Budapest, Hungary, dLaboratory of

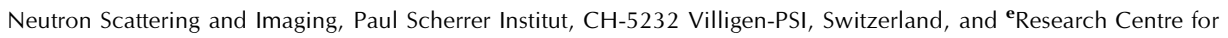
Natural Sciences, Hungarian Academy of Sciences, Magyar Tudósok körútja 2, Budapest, Hungary. ${ }^{*}$ Correspondence e-mail: tianqiang@yahoo.com, almasy@mail.kfki.hu
\end{abstract}

A series of calibration measurements have been performed on Suanni - the first small-angle neutron scattering spectrometer entering into routine user operation in China - aiming to characterize instrument performance and to provide a reference for user experiments. Various calibration standards were used to verify the precise association of the scattering intensity $I$ with the scattering vector magnitude $q$ at short and medium sample-detector distances. The measurements recorded for silver behenate powder revealed a slight shift of the neutron wavelength $(\lambda)$ at the sample position as compared to the nominal $\lambda$ calculated by the selector constant. The deviation was more significant with $\lambda>$ $0.8 \mathrm{~nm}$ owing to the shape of the incoming neutron spectrum incident on the selector. The measured dead time for the entire detection system is $2.7 \mu \mathrm{s}$, as determined by measurements with varying fluxes. A protonated/deuterated polystyrene blend was employed as the primary standard to calibrate the secondary standard $\mathrm{H}_{2} \mathrm{O}$. The instrument covers an effective $q$ range between 0.013 and $5 \mathrm{~nm}^{-1}$, as demonstrated by scattering curves obtained from a monodisperse poly(methyl methacrylate) nanoparticle suspension and a glassy carbon plate.

\section{Introduction}

Suanni, the first routinely employed small-angle neutron scattering (SANS) instrument located at Mianyang Research Reactor in China, was commissioned in 2014 (Peng et al., 2016) and has been in routine use since 2015, operating about 120 days annually. After a few years of operation, a variety of calibration work has been performed on Suanni, which has provided detailed data on instrument performance and accuracy.

On a conventional SANS instrument operating on a continuous neutron source the samples are illuminated with a quasi-monochromatic neutron beam and the scattered intensity is recorded as a function of scattering angle. The calibration of a SANS instrument consists of the development and parametrization of the algorithm expressing the raw scattering data as absolute scattering cross section of the sample as a function of the scattering vector magnitude $q$. To this end, 
several tasks need to be performed, such as the determination of the wavelength and wavelength distribution of the incoming neutrons, the evaluation of the mathematical functions used for conversion and manipulation of the raw data, and the conversion of the measured intensity data to the absolute intensity scale.

Accurate information about the neutron wavelength is essential for the evaluation of the dimension parameters of the investigated samples. At Suanni, as at most SANS instruments based on reactor sources, a nearly monochromatic neutron beam is formed by a neutron velocity selector. The value of the mean wavelength can be calculated from the mechanical characteristics of the selector, determined by the time-of-flight method or by measuring known standard samples at one or a few selector speeds. A possible source of error in the neutron wavelength can be the assumption that it is inversely proportional to the selector rotation speed for all wavelengths. In practice, the wavelength of neutrons passing the selector does not necessarily coincide with the nominal wavelength calculated as the selector constant divided by the rotation speed, and thus should be determined independently.

Scaling the measured intensity into absolute units, typically in units of $\mathrm{cm}^{-1}$, is the basis for determination of several important parameters of the sample, such as the number density of scatterers, their volume fraction and the interfacial area. In addition, presenting the data on absolute scale offers a valuable diagnostic tool in SANS experiments (Wignall \& Bates, 1987), allowing users to relate the discrepancies between the observed and calculated intensities to potential artefacts (Schelten et al., 1977), to new physical processes (Bates \& Wignall, 1986) or to the characteristics of the studied samples. Despite the advantages of using absolute intensity, users are often not willing to perform calibration procedures, especially measuring weakly scattering standard samples (e.g. $\mathrm{H}_{2} \mathrm{O}$, at longer detector distances) because of the limited beam time. The acquisition of absolute calibrated data for Suanni can be facilitated by the provision of a fast calibration method using strong scattering samples which would allow users to perform reference measurements within $30 \mathrm{~min}$ - a minor fraction of the typically granted 1-2 days of beam time.

In the past few decades several reports have described various aspects of the aforementioned tasks and have proposed increasingly accurate solutions for the specific problems related to handling of the various small deviations from the geometry of an idealized SANS instrument (Gilles $e t$ al., 1998; Strunz et al., 2000, 2004; Almásy et al., 2006; Brûlet et al., 2007; Karge et al., 2017). Many of these effects can be regarded as second-order corrections and were largely ignored in the past; and they are still ignored by most instrument users in the present, particularly when the correction procedures are not integrated into the data processing software.

In the present work, the calibration of the wavelength, the scattering vector magnitude $q$ and the absolute intensity on Suanni have been performed and verified by measuring various standard samples. These benchmark measurements and data not only provide an informative reference for users but are indispensable for the characterization of the instru- ment performance and give a valuable diagnostic tool for the instrument scientist.

\section{Experimental}

The silver behenate $\left[\mathrm{CH}_{3}\left(\mathrm{CH}_{2}\right)_{20} \mathrm{COOAg}\right.$, denoted here as $\mathrm{AgBE}]$ used in this work was produced by Alfa Aesar with a lot number of E15T020. Mesostructured MSU-H (hexagonal) and MSU-F (cellular foam) silica, in which MSU is the abbreviation of Michigan State University, were purchased from Sigma-Aldrich Co. (lot Nos. MKBS4565V and MKBX5986V, respectively). All the powders were loaded into Hellma quartz cells of $2 \mathrm{~mm}$ light path. Deuterated polystyrene (d-PS) with a weight-average molecular weight $\left(M_{\mathrm{w}}\right)$ of 236500 and a number-average molecular weight $\left(M_{\mathrm{N}}\right)$ of $215000\left(M_{\mathrm{w}} / M_{\mathrm{N}}=1.10\right)$ and protonated polystyrene (h-PS) with $M_{\mathrm{w}}$ of 290000 and $M_{\mathrm{N}}$ of $273000\left(M_{\mathrm{w}} / M_{\mathrm{N}}=1.06\right)$ were purchased from Polymer Source and Alfa Aesar, respectively. An h-PS/d-PS blend was prepared by mixing h-PS and d-PS with equal mass ratio in toluene solution, and then evaporating the solvent at room temperature. Glassy carbon plates were procured from Alfa Aesar (lot No. J29Z017). Fullerene C60 and C70 were obtained from Sigma-Aldrich Co. (lot Nos. MKBJ4461V and MKBV3813V, respectively). Nearly monodisperse poly(methyl methacrylate) (PMMA) spherical nanoparticles were provided by the Institute of Chemistry, Chinese Academy of Sciences. Before measurements, the asobtained PMMA nanoparticles $\left(22 \mathrm{mg} \mathrm{ml}^{-1}\right)$ suspended in $\mathrm{H}_{2} \mathrm{O}$ were diluted with $\mathrm{D}_{2} \mathrm{O}$ in order to increase the contrast and decrease the incoherent scattering. The final ratio of $\mathrm{D}_{2} \mathrm{O}$ and $\mathrm{H}_{2} \mathrm{O}$ in the medium was $4: 1$ and the nanoparticle concentration was $0.4 \mathrm{mg} \mathrm{ml}^{-1}$. The morphology of the PMMA nanoparticles was examined by using a Hitachi s-4300 field emission scanning electron microscope.

The SANS measurements were performed on the Suanni spectrometer at the China Mianyang Research Reactor. The scattering intensity $I(q)$ was recorded as a function of scattering vector magnitude $q=4 \pi \sin \theta / \lambda$, where $\lambda$ is the wavelength of the incident neutrons and $\theta$ is half of the scattering angle. A helical mechanical velocity selector produced a quasimonochromatic neutron beam with wavelengths between 0.35 and $1.6 \mathrm{~nm}$ and with a nominal wavelength resolution $(\Delta \lambda / \lambda)$ of $18 \%$. The scattered neutrons were recorded by a ${ }^{3} \mathrm{He}$ gasfilled two-dimensional multiwire proportional counter (MWPC) having $128 \times 128$ pixels and a pixel size of $5.4 \times$ $5.4 \mathrm{~mm}$. The transmission was calculated as the ratio of the attenuated direct beam intensities with and without the sample. The scattering data were processed using the BerSANS software (Keiderling, 2002). The data reduction algorithm included subtraction of air and quartz cell scattering from the raw measured data and corrections for transmission, sample thickness and detector efficiency. The least-squares fitting was performed with the SASfit software (Breßler et al., 2015). In order to cross-check the obtained results from Suanni, some of the samples were measured with the CREDO SAXS instrument at the Research Centre for Natural Sciences (Hungary), SANS-2 at the Paul Scherrer Institute (PSI, 
Switzerland) and NG-7 at the National Institute of Standards and Technology (NIST, USA).

\section{Results and discussion}

\subsection{Wavelength calibration}

Previously, a multiblade rotor neutron velocity selector (MBR-7-420-360, Mirrotron Ltd, Hungary) (Rosta et al., 2006) was installed at the Suanni instrument, providing a relatively high wavelength resolution $(\Delta \lambda / \lambda=5-12 \%)$. Since March 2017, a new helical mechanical neutron velocity selector (NVS071, Airbus DS GmbH, Germany) has been in operation at Suanni, resulting in a gain of 4 times neutron flux compared with the original selector when the latter is set to $\Delta \lambda / \lambda=10 \%$. However, the gain has come at the expense of reduced wavelength resolution $(\Delta \lambda / \lambda=18 \%)$. The two selectors are used interchangeably when one of them is under maintenance. The calibration of the previous multiblade selector was recently reported (Peng et al., 2016). Here we present calibration measurements performed exclusively with the new NVS071 selector.

The intensity spectrum was determined by measuring the count rate of the direct beam on the detector (Fig. 1), revealing a maximum intensity at a velocity selector rotation speed of $18700 \mathrm{r} \mathrm{min}^{-1}$, corresponding to wavelength of $0.53 \mathrm{~nm}$ as calculated from the selector constant. Therefore, this wavelength is frequently chosen for sample measurements. Using three sample-to-detector (SD) distances, namely, $1.15,5.25$ and $10.25 \mathrm{~m}$, an effective $q$ window of $0.04-3.5 \mathrm{~nm}^{-1}$ can be covered at the fixed wavelength $\lambda=0.53 \mathrm{~nm}$. At this wavelength, with a circular source and sample apertures of 30 and $6 \mathrm{~mm}$ in diameter, with all the neutron guides in the beam, and with rectangular diaphragms $(30 \times 100 \mathrm{~mm})$ between guides, the maximum neutron flux measured by the fission chamber under a reactor power of $20 \mathrm{MW}$ at the sample position is $1 \times 10^{7} \mathrm{~cm}^{-2} \mathrm{~s}^{-1}$.

The wavelength was calibrated by the scattering from $\mathrm{AgBE}$ recorded at different rotation speeds at the same SD distance of $1.15 \mathrm{~m}$. The distance $\left(L_{\mathrm{WD}}\right)$ from the sapphire

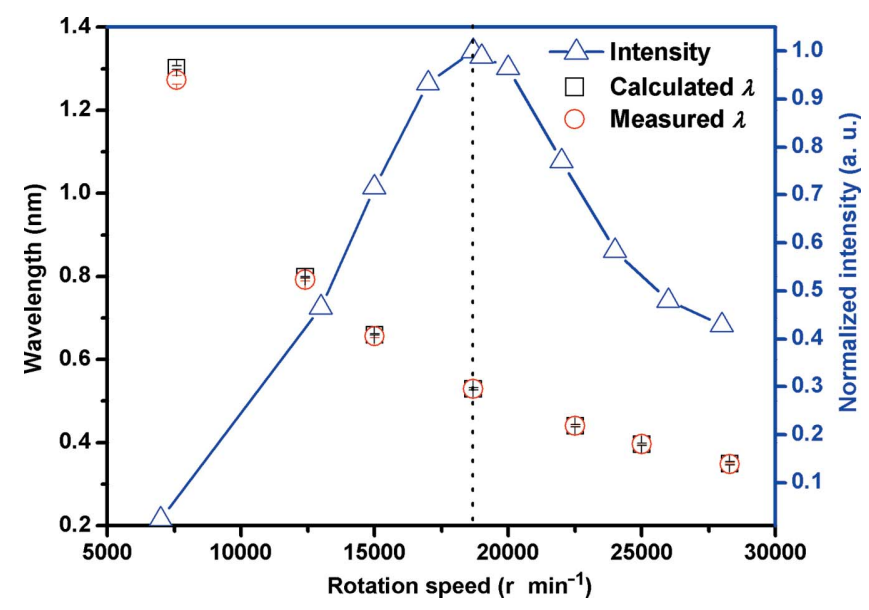

Figure 1

The wavelength calibration for Suanni using the NVS071 selector. window in the front of the detector tank to the surface of the two-dimensional detector was measured by a laser rangefinder, and the distance $\left(L_{\mathrm{SW}}\right)$ between the sample and the sapphire window was measured by a metre rule. The SD distance was also offset by the mean detection path $L_{\mathrm{DP}}$ inside the two-dimensional detector. For Suanni, $L_{\mathrm{DP}}=3.4 \mathrm{~cm}$ at $\lambda=$ $0.53 \mathrm{~nm}$. This is determined by measuring the scattering from $\mathrm{AgBE}$ at two SD distances, enabling a precise calculation of the Bragg angle $2 \theta$ by $\tan (2 \theta)=\Delta r / \Delta L$, where $\Delta r$ and $\Delta L$ are the difference values of the peak positions and SD distances (Henderson, 1997; Strunz et al., 2004). The total SD distance is equal to the sum of $L_{\mathrm{SW}}, L_{\mathrm{WD}}$ and $L_{\mathrm{DP}}$ (Fig. 2). Note that the detection path is dependent on the neutron wavelength, efficiency of the detector and scattering angle. A larger neutron wavelength and higher scattering angle may result in a shorter $L_{\mathrm{DP}}$. The measured wavelength $\lambda_{\mathrm{m}}$ was calculated from $\mathrm{AgBE}$ measurements via the Bragg equation, $\lambda_{\mathrm{m}}=2 d_{100} \sin \theta^{*}$, where $d_{100}$ is the long-period spacing of $\mathrm{AgBE}, 5.838 \mathrm{~nm}$, and $\theta^{*}$ is determined by Gaussian function fitting to the 001 peak of the $I(\theta)$ scattering curve. Traditionally, the calculated wavelength $\left(\lambda_{c}\right)$ at a given speed is calculated as $C / \omega$, where $C$ is a constant determined by calibration and $\omega$ is the selector rotation speed. In the present test, $\lambda_{\mathrm{m}}$ and $\lambda_{\mathrm{c}}$ match each other well in the wavelength range of $0.35-0.66 \mathrm{~nm}$ (Fig. 1). The difference between $\lambda_{\mathrm{m}}$ and $\lambda_{\mathrm{c}}$ becomes larger with increasing wavelength, reaching $2.4 \%$ at $\lambda_{c}=1.3 \mathrm{~nm}$, while remaining below $1 \%$ for wavelength values smaller than $0.8 \mathrm{~nm}$. This difference between the measured and calculated values can be attributed to the pronounced decrease of the incoming neutron flux with decreasing neutron wavelength in this region. The neutron intensity measured at the sample position is about 40 times lower at the wavelength of $1.3 \mathrm{~nm}$ than at $0.53 \mathrm{~nm}$. Almásy et al. (2006) reported a similar wavelength shift at longer wavelengths for a multidisc-type velocity selector on the 'Yellow Submarine' SANS spectrometer at Budapest Neutron Centre, Hungary, and derived an analytical correction formula based on the shape of the intensity distribution in the incoming neutron beam.

To obtain size parameters with precision as high as $1 \%, \lambda_{\mathrm{m}}$ is preferred, especially for longer-wavelength measurements.

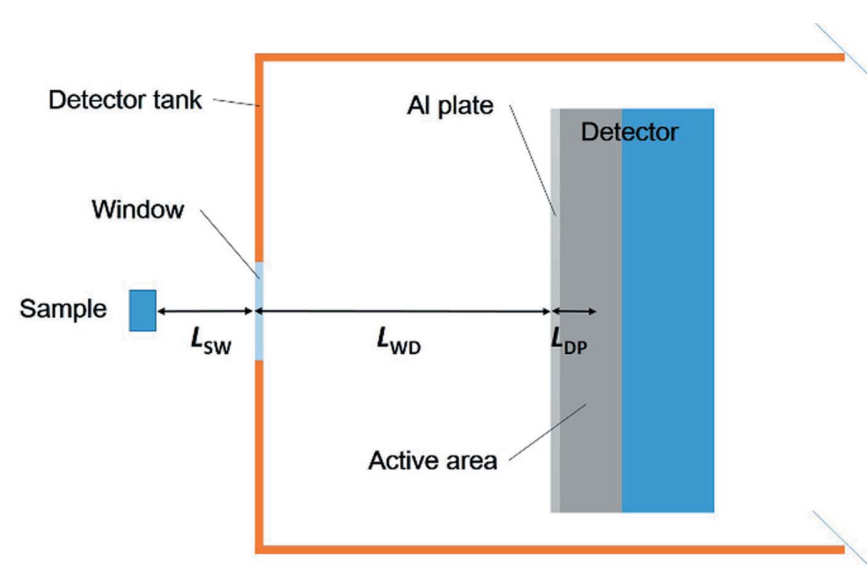

Figure 2

Schematic representation of the total SD distance. 
Table 1

Geometry resolution parameters for the normal setups of Suanni.

\begin{tabular}{llllllllll}
\hline$L_{1}(\mathrm{~mm})$ & $L_{2}(\mathrm{~mm})$ & $R_{1}(\mathrm{~mm})$ & $R_{2}(\mathrm{~mm})$ & $x_{3}(\mathrm{~mm})$ & $\lambda(\mathrm{nm})$ & $\sigma_{2 \theta}^{2}$ & {$\left[\sigma_{q}^{2}\right]_{\text {geo }}\left(\mathrm{nm}^{-2}\right)$} & Calculated $\sigma_{2 \theta}\left({ }^{\circ}\right)$ & Measured $\sigma_{2 \theta}\left({ }^{\circ}\right)$ \\
6900 & 1500 & 15 & 4 & 5.4 & 0.53 & $9.79 \times 10^{-6}$ & $1.37 \times 10^{-3}$ & 0.179 & 0.174 \\
6900 & 5200 & 15 & 4 & 5.4 & 0.53 & $3.45 \times 10^{-6}$ & $4.85 \times 10^{-4}$ & 0.107 & 0.085 \\
12900 & 10200 & 15 & 4 & 5.4 & 0.53 & $9.69 \times 10^{-7}$ & $1.36 \times 10^{-4}$ & 0.056 & 0.048 \\
12900 & 10200 & 15 & 4 & 5.4 & 0.85 & $9.69 \times 10^{-7}$ & $5.29 \times 10^{-5}$ & 0.056 & - \\
\hline
\end{tabular}

Using a velocity selector setting with narrower wavelength spread would diminish the difference between the calculated and measured wavelength.

\section{2. $q$ calibration}

3.2.1. $q$ resolution. The variance in $q$ is the sum of squared components of the geometry and wavelength resolutions, given by

$$
\sigma_{q}^{2}=\left\langle(q-\langle q\rangle)^{2}\right\rangle=\left(\frac{4 \pi}{\lambda}\right)^{2} \sigma_{\theta}^{2}+q^{2} \frac{\sigma_{\lambda}^{2}}{\lambda^{2}}
$$

where the first term in the summation corresponds to the geometric resolution of the instrument at a specific setup and the second term to the $q$-dependent component. $\sigma_{\theta}$ and $\sigma_{\lambda}$ are the variance in $\theta$ and $\lambda$, respectively. The physical meaning of the geometry resolution is the divergence of the direct beam at $q=0$. The contribution in the horizontal direction is expressed as

$$
\left[\sigma_{q x}^{2}\right]_{\text {geo }}=\left(\frac{2 \pi}{\lambda}\right)^{2}\left[\frac{R_{1}^{2}}{4 L_{1}^{2}}+\left(\frac{L_{2}+L_{1}}{L_{2} L_{1}}\right)^{2} \frac{R_{2}^{2}}{4}+\frac{x_{3}^{2}}{12 L_{2}^{2}}\right],
$$

where $L_{1}$ is the collimation length, $L_{2}$ the SD distance, $R_{1}$ the radius of the source aperture, $R_{2}$ the radius of the sample aperture and $x_{3}$ the pixel size in the horizontal direction. Considering the effect of gravity on the neutron trajectories, the geometry resolution in the vertical direction is given by

$$
\begin{aligned}
{\left[\sigma_{\text {qy }}^{2}\right]_{\text {geo }}=} & \left(\frac{2 \pi}{\lambda}\right)^{2}\left[\frac{R_{1}^{2}}{4 L_{1}^{2}}+\left(\frac{L_{2}+L_{1}}{L_{2} L_{1}}\right)^{2} \frac{R_{2}^{2}}{4}+\frac{y_{3}^{2}}{12 L_{2}^{2}}\right. \\
& \left.+A^{2}\left(\left\langle\lambda^{4}\right\rangle-\left\langle\lambda^{2}\right\rangle^{2}\right)\right],
\end{aligned}
$$

where $A=L_{2}\left(L_{1}+L_{2}\right) g m^{2} /\left(2 h^{2}\right), g$ is the acceleration due to gravity, $m$ the neutron mass, $h$ Planck's constant and $y_{3}$ the pixel size in the vertical direction (Hammouda \& Mildner, 2007; Hammouda, 2016). Although the effect of gravity is stronger for longer wavelengths, the contribution of gravitational effects to the geometry resolution in the vertical direction is less than $1 \%$ at the longest collimation and SD distance with $\lambda<0.85 \mathrm{~nm}$ for Suanni. Therefore, the geometry resolution at any azimuthal angle can be approximated as

$$
\begin{aligned}
{\left[\sigma_{q}^{2}\right]_{\mathrm{geo}} } & =\sigma_{q}^{2}(q=0)=\left(\frac{4 \pi}{\lambda}\right)^{2} \sigma_{\theta}^{2}=\left(\frac{2 \pi}{\lambda}\right)^{2} \sigma_{2 \theta}^{2} \\
& =\left(\frac{2 \pi}{\lambda}\right)^{2}\left[\frac{R_{1}^{2}}{2 L_{1}^{2}}+\left(\frac{L_{2}+L_{1}}{L_{2} L_{1}}\right)^{2} \frac{R_{2}^{2}}{2}+\frac{x_{3}^{2}}{6 L_{2}^{2}}\right],
\end{aligned}
$$

where $x_{3}=y_{3}$. The frequently chosen configuration setups on Suanni are $L_{2}=1.5,5.2$ and $10.2 \mathrm{~m}$, while $L_{1}$ is fixed at $6.9 \mathrm{~m}$ for the short and medium $L_{2}$, and $12.9 \mathrm{~m}$ for the longest $L_{2}$. The geometry resolution improves with increasing collimation length and wavelength (Table 1). The measured direct beam width at the normal setups for Suanni is shown in Fig. 3. It is assumed that the direct beam profile conforms to a Gaussian distribution, and the fitted beam widths (denoted as measured $\left.\sigma_{2 \theta}\right)$ are shown in the last column in Table 1 ; these are fairly close to the calculated $\sigma_{2 \theta}$ values.

Assuming that the wavelength distribution of the beam transmitted by the mechanical velocity selector follows a Gaussian function, the $q$-dependent component in equation (1) can be expressed as

$$
\left[\sigma_{q}^{2}\right]_{\lambda}=\frac{1}{8 \ln 2}\left(\frac{\Delta \lambda}{\lambda}\right)^{2} q^{2}
$$

where $\Delta \lambda$ is the FWHM of the Gaussian function. The relative $q$ resolution $\left(\sigma_{q} / q\right)$ as a function of $q$ can be obtained by combining the components of the geometry and wavelength resolution. The resolution curves for the typical configurations are shown in Fig. 4. The value of $\sigma_{q} / q$ near and far away from the beamstop is dominated by the geometry and wavelength resolution, respectively. Usually, for quasi-monochromatic

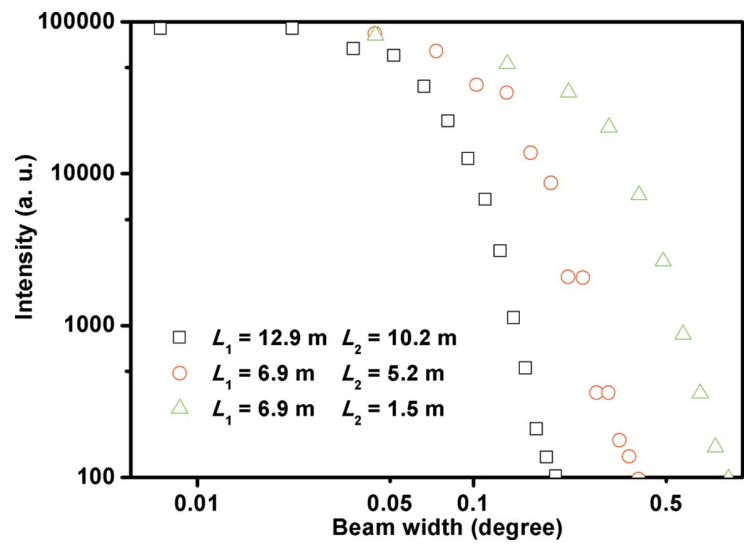

Figure 3

The beam width of Suanni measured at different collimation lengths $\left(L_{1}\right)$ and SD distances $\left(L_{2}\right)$ with sample and source apertures of 4 and $15 \mathrm{~mm}$ in radius. 
Table 2

Fitted peak positions $\left(\mathrm{nm}^{-1}\right)$ for the SAS data of AgBE, MSU-F and MSU-H.

\begin{tabular}{llll}
\hline Sample & Suanni SANS & CREDO SAXS & SANS-2 at PSI \\
\hline AgBE & $1.0745(7)$ & $1.0730 \pm(4)$ & - \\
MSU-F & $0.3209(7)$ & - & $0.3182(3)$ \\
MSU-H & $0.6033(3)$ & - & $0.5968(2)$ \\
\hline
\end{tabular}

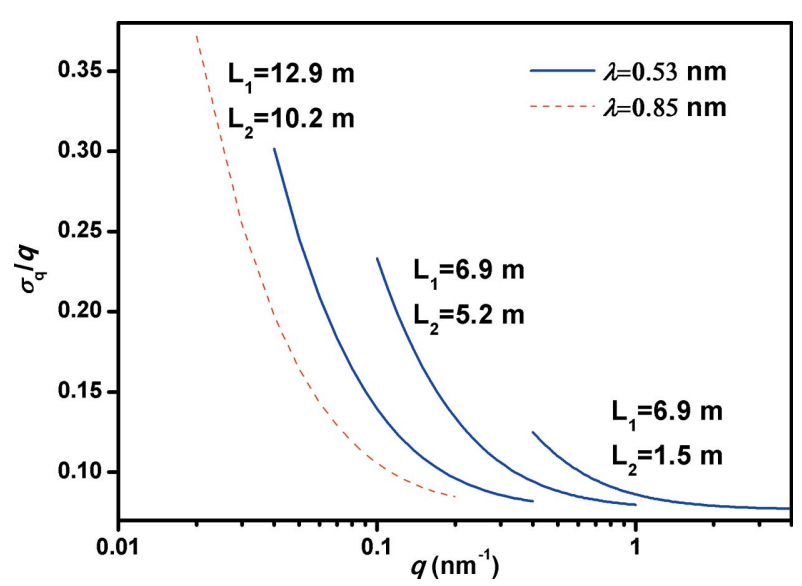

Figure 4

Relative $q$ resolution as a function of $q$ calculated for various instrument configurations.

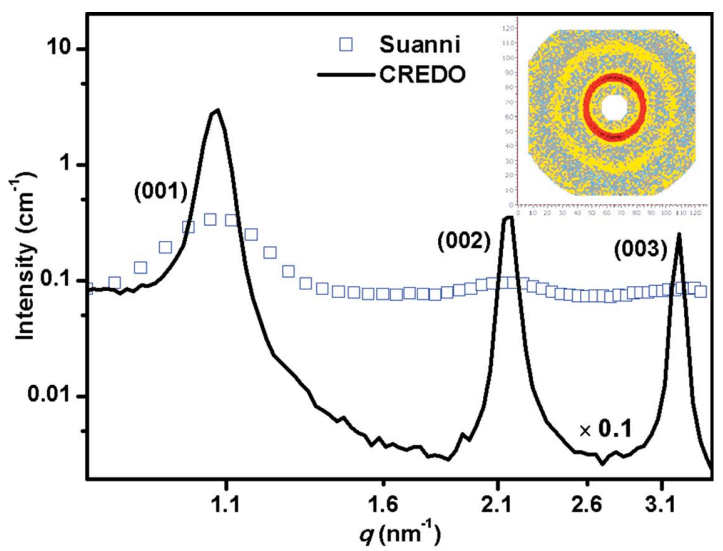

(a)

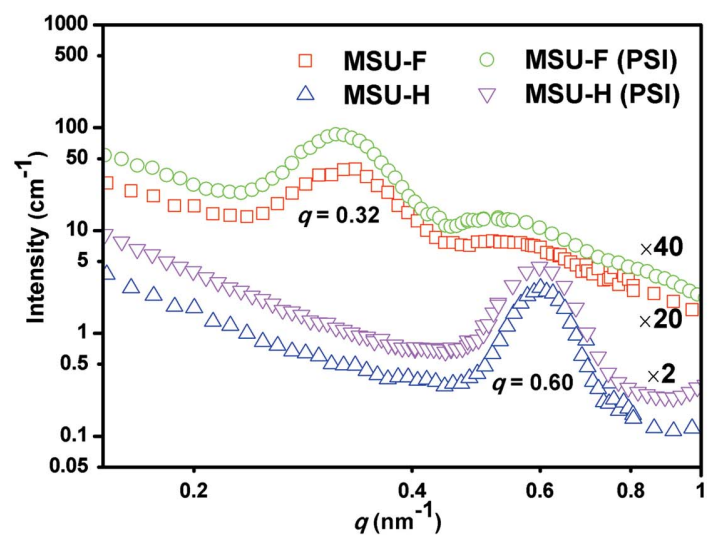

(b)

Figure 5

SANS scattering curves obtained from $\operatorname{AgBE}(a)$ and from MSU-H and MSU-F mesoporous silica $(b)$. The solid line corresponds to the SAXS data of $\mathrm{AgBE}$ measured by CREDO.
SANS instruments, for optimizing the resolution at selected parts of the scattering curves - exhibiting peaks, local minima or inflection points - the configuration should be chosen so that these parts of the scattering curve are measured closer to the edge of the detector.

3.2.2. Calibration at medium and high $q$. AgBE with its first Bragg peak centred at $q_{\mathrm{pk}}=1.076 \mathrm{~nm}^{-1}\left(d_{100}=5.838 \mathrm{~nm}\right)$ is the most frequently chosen standard for $q$ calibration of SANS instruments (Gilles et al., 1998; Nyam-Osor et al., 2012; Wacha et al., 2014). It is good practice to measure such a sample at least once during each user experiment, in order to confirm the wavelength and SD distance, and occasionally to estimate the resolution smearing of the scattering patterns of the measured samples. The two-dimensional pattern and $I(q)$ curves of $\mathrm{AgBE}$ are shown in Fig. 5(a), featuring the 001, 002 and 003 diffraction peaks. The FWHM of the first Bragg peak derived from Gaussian fitting to SANS data is $0.23 \mathrm{~nm}^{-1}$, which is much higher than that $\left(0.06 \mathrm{~nm}^{-1}\right)$ of the SAXS data. Nevertheless, it is still suitable for determining the wavelength from the first neutron Bragg peak (Gilles et al., 1998; Keiderling et al., 1999). The first diffraction peak of AgBE is fitted by a Gaussian function convoluted with the resolution function. As shown in Table 2, the deviation in peak positions between the data measured by Suanni and CREDO is $0.2 \%$.

The limitation of using $\mathrm{AgBE}$ is that the $q$ range of interest is often lower than the first diffraction peak of AgBE. Therefore, we propose here to use the commercially available MSU-F and MSU-H ordered mesoporous silica with cell parameters around 22 and $11 \mathrm{~nm}$ as calibration standards (Fig. $5 b$ ) for $q$ calibration at smaller $q$ ranges, i.e. at longer SD distances $\left(L_{2}>4 \mathrm{~m}\right)$. Note that the diffraction peak positions of MSU-F and MSU-H are dependent on sample preparation, and therefore each sample should be calibrated independently. In the present case, knowing the wavelength and the detector distances, the $q$ values of the peak positions can be accurately determined and the samples used as easy standards in routine user experiments. A comparative measurement on the same batch of MSU-F and MSU-H conducted on the SANS-2 instrument at PSI (Fig. $5 b$ and Table 2) indicates the overall agreement of the calibration.

\subsection{Absolute intensity calibration}

3.3.1. Dead-time evaluation of the Suanni detector. Any complex detection system with electronic components needs a certain time to record and process discrete events, during which the system is not able to record another event. For recording neutrons with an MWPC detector, the two important sources of dead time are the physical inactivity of the local area of the detector after a neutron hit and the time needed for the detection and positioning of the neutron by the electronics. In the used MK-640N-1 two-dimensional MWPC detector the first events in each channel are read out every $200 \mathrm{~ns}$, independently from the occurrence of any further event in this time period, resulting in a non-paralysable mode of operation of the detector electronics. 
The diminished counting rate resulting from dead time may cause inaccuracies in the following cases: comparing samples with significantly different count rates; merging data that are measured at different $\mathrm{SD}$; and absolute scaling when the sample and the calibration standard scatter neutrons with very different count rates. Therefore, prior to data reduction and absolute intensity calibration, the dead time should be determined.

In general, the counting efficiency related to dead-time losses can be described by a non-paralysable or paralysable model. For low count rates and small dead-time losses, the two models give similar predictions (Knoll, 2010). Assuming a non-paralysable detection system for Suanni, the measured count rate $N_{\mathrm{M}}$ is described as (Lindner \& Zemb, 2002)

$$
N_{\mathrm{M}}=\frac{N_{\mathrm{T}}}{1+N_{\mathrm{T}} \tau},
$$

where $\tau$ is the dead time and $N_{\mathrm{T}}$ is the true count rate. For a series of transmission measurements (the beamstop is out of the beam) with different circular apertures and collimation lengths for the same sample at an SD distance of $5.2 \mathrm{~m}$, the ratio is derived as

$$
N_{\mathrm{M}} / N_{\mathrm{ME}}=T+(1-T) \tau N_{\mathrm{M}},
$$

where $N_{\mathrm{ME}}$ is the measured count rate with no sample and $T$ is the transmission of the sample. Plotting $N_{\mathrm{M}} / N_{\mathrm{ME}}$ as a function of $N_{\mathrm{M}}$ yields a linear behaviour with intercept $T$ and slope $k$. Then the dead time can be estimated as $\tau=k /(1-T)$. The measured results for a $1 \mathrm{~mm}$-thick $\mathrm{H}_{2} \mathrm{O}$ sample filled into a $20 \mathrm{~mm}$-width quartz Hellma cell are shown in Fig. 6. The calculated $\tau$ is equal to $2.7 \mu \mathrm{s}$, which is the dead time for the entire detection system. On the basis of the estimated dead time, the relative loss factor is given by

$$
\text { Loss }=\frac{N_{\mathrm{T}}-N_{\mathrm{M}}}{N_{\mathrm{T}}}=\frac{N_{\mathrm{T}} \tau}{1+N_{\mathrm{T}} \tau} .
$$

The loss factor becomes larger than $1 \%$ when the total count rates are higher than 4000 counts per second. Usually, the count rate of samples is smaller than 1000 counts per second, and the dead-time correction can be ignored. Nevertheless, for

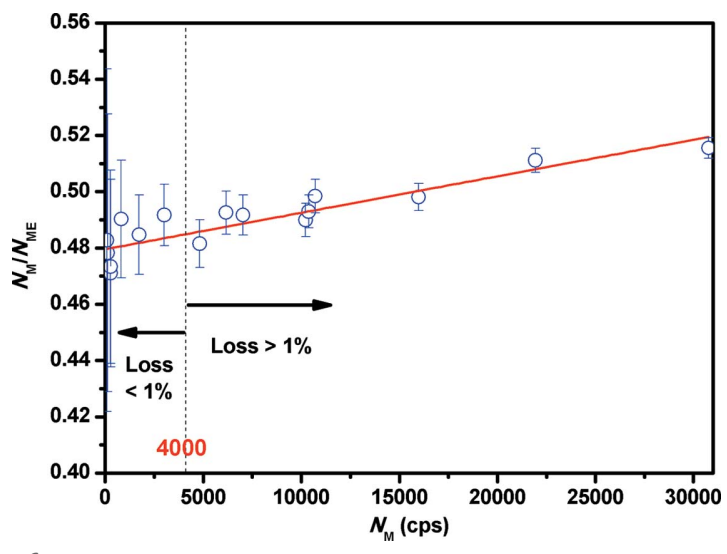

Figure 6

Dead-time evaluation by the attenuation method. precise data reduction, it is always recommended to correct for dead-time losses.

3.3.2. Absolute intensity calibration. Absolute calibration can be performed either by the attenuated direct beam method (Lindner \& Zemb, 2002; Dewhurst et al., 2016) or by using standard samples (Glinka et al., 1998) with known differential cross section. Formulas for the macroscopic cross section (in units of $\mathrm{cm}^{-1}$ ) for the two methods are written as

$$
\begin{gathered}
{\left[\frac{\mathrm{d} \Sigma(q)}{\mathrm{d} \Omega}\right]_{\mathrm{s}}=\left(\frac{I_{\mathrm{s}}}{I_{\text {direct }}}\right)\left(\frac{T_{\text {atten }}}{d_{\mathrm{s}}}\right)\left(\frac{1}{\Delta \Omega}\right),} \\
{\left[\frac{\mathrm{d} \Sigma(q)}{\mathrm{d} \Omega}\right]_{\mathrm{s}}=\left[\frac{I_{\mathrm{s}}}{I(q=0)_{\mathrm{std}}}\right]\left(\frac{d_{\mathrm{std}}}{d_{\mathrm{s}}}\right)\left[\frac{\mathrm{d} \Sigma(q=0)}{\mathrm{d} \Omega}\right]_{\mathrm{std}},}
\end{gathered}
$$

where $I_{\mathrm{s}}$ is the scattering intensity of the sample, corrected for background, transmission, detector efficiency and dead-time losses of the detector, expressed in counts per pixel per second; $d_{\text {std }}$ and $d_{\mathrm{s}}$ are the thickness (in units of $\mathrm{cm}$ ) of the sample and standard sample; $T_{\text {atten }}$ is the transmission of the attenuator; $\Delta \Omega$ is the solid angle covered by one pixel of the detector; $I(q=0)_{\text {std }}$ is the extrapolation of the measured intensity of a standard sample to $q \rightarrow 0$; and $[\mathrm{d} \Sigma(q=0) / \mathrm{d} \Omega]_{\text {std }}$ is the theoretical macroscopic cross section of a standard sample at $q=0$. For the attenuated direct beam method, the incoming beam intensity is calculated as the direct beam intensity $I_{\text {direct }}$ over $T_{\text {atten }}$. With the help of NIST macros for Igor Pro (https://www.wavemetrics.com/), the scattering data from Suanni can be put on an absolute scale.

The standard sample method is employed to calibrate the absolute intensity on Suanni. Light water $\left(\mathrm{H}_{2} \mathrm{O}\right)$ is commonly used for absolute intensity calibrations of SANS measurements because it is readily available at all laboratories and its scattering is mainly incoherent. However, owing to inelastic and multiple scattering effects, $\mathrm{H}_{2} \mathrm{O}$ should only be used as a secondary standard and must be pre-calibrated at each individual instrument. Lindner (2000) calibrated a water sample against dilute PS solution for D11 and D22 at ILL. Herein, an h-PS/d-PS blend with much stronger scattering is used as the first standard to calibrate the $1 \mathrm{~mm}$-thick $\mathrm{H}_{2} \mathrm{O}$. The $g$ factor is calculated from

$$
\begin{gathered}
g=\lim _{q \rightarrow 0}\left(I^{\text {calcuated }} / I^{\text {measured }}\right)=x(1-x)\left(b_{\mathrm{H}}-b_{\mathrm{D}}\right)^{2} n Z^{2} / I_{0}^{\text {measured }}, \\
I(q)^{\text {measured }}=I_{0}^{\text {measured }} P(q), \\
P(q)=\left(2 / U^{2}\right)[U+\exp (-U)-1] \text { with } U=q^{2} R_{\mathrm{g}}^{2},
\end{gathered}
$$

where $I(q)^{\text {measured }}$ is the intensity of h-PS/d-PS calibrated by $1 \mathrm{~mm}$-thick $\mathrm{H}_{2} \mathrm{O}$ without considering the $g$ factor, $x=0.48$ is the volume fraction of d-PS, $b_{\mathrm{H}}$ and $b_{\mathrm{D}}$ are the coherent scattering lengths of $\mathrm{C}_{8} \mathrm{H}_{8}$ and $\mathrm{C}_{8} \mathrm{D}_{8}$ units, $n$ is the number of molecules per unit volume, $P(q)$ is the form factor of a Gaussian chain, $Z$ is the polymerization index, and $R_{\mathrm{g}}$ is the radius of gyration. The calculated $g$ factor is 1.35 (Fig. 7a). The $g$ factor is instrument specific and $\lambda$ dependent, and therefore this value is only valid under the defined conditions, i.e. 
$\lambda=0.53 \mathrm{~nm}, L_{1}=6.9 \mathrm{~m}, L_{2}=5.2 \mathrm{~m}, T=298 \mathrm{~K}, d=1 \mathrm{~mm}, \mathrm{MK}-$ $640 \mathrm{~N}-1 \mathrm{MWPC}$ detector (Mirrotron, $2.1 \mathrm{bar}^{3} \mathrm{He}$ and $0.7 \mathrm{bar}$ $\mathrm{CF}_{4} ; 1$ bar $\left.=10^{5} \mathrm{~Pa}\right)$.

Glassy carbon, featuring a long plateau on its scattering curve, is a very popular secondary standard (Zhang et al., 2010; Wacha et al., 2014; Allen et al., 2017). When the intensity of glassy carbon calibrated with $\mathrm{H}_{2} \mathrm{O}$ is multiplied by the abovedetermined $g$ factor, the final calibrated scattering curve almost overlaps with the data measured by the NG-7 instrument at NIST (Fig. 7b). The same piece of glassy carbon was used in both measurements, and the average deviation ratio is about $3 \%$ over the $q$ range of $0.1-1 \mathrm{~nm}^{-1}$. This indicates that the used procedure was sufficiently accurate for absolute intensity calibration at the given wavelength.

3.3.3. Accessible $q$ range and scattering cross sections at Suanni. A knowledge of the effective upper and lower $q$ limits for the SANS instrument is essential for estimating the feasibility and planning of specific experiments in advance. The accessible $q$ range calculated from the geometry parameters of Suanni and the incident wavelength is shown in Fig. 8. An order of magnitude of $q$ range can be covered in one setup. Usually, in the high- $q$ range, the coherent scattering intensity of the sample becomes very weak and comparable to or lower than the incoherent background of the sample and

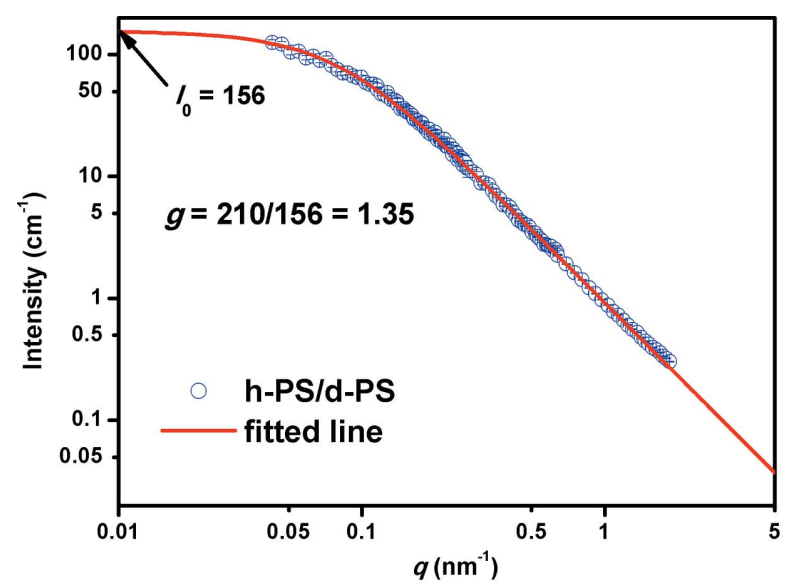

(a)

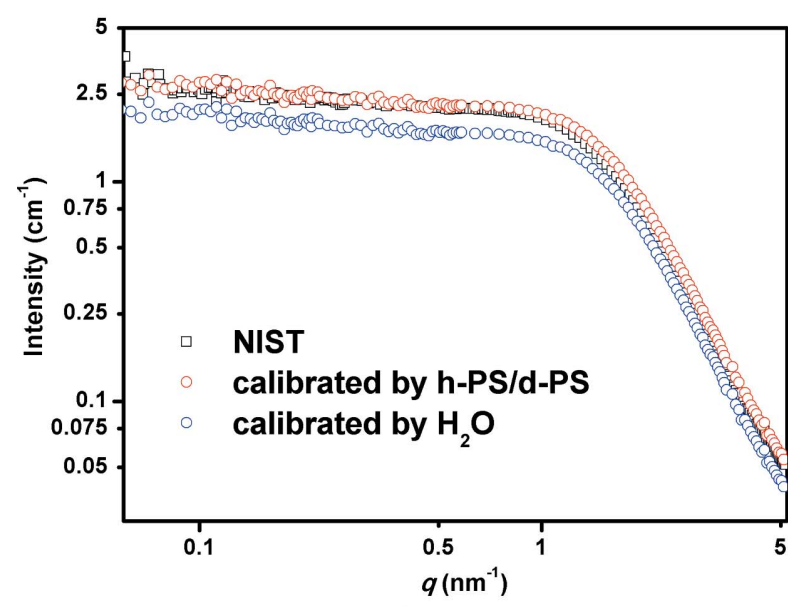

(b)

Figure 7

SANS curves of h-PS/d-PS ( $a$ ) and glassy carbon calibrated by $\mathrm{H}_{2} \mathrm{O}(b)$. instrument environment background. It is instructive, then, to provide data on the sensitivity of a given type of experiment. In the high- $q$ range, the sensitivity due to the signal-tobackground ratio is the usual limiting factor, while in the low- $q$ range, the useful information is the practically attainable smallest $q$ value for various scattering intensities.

To demonstrate the lowest measurable intensities, we recorded scattering from fullerene suspensions $\left(\sim 5 \mathrm{mg} \mathrm{ml}^{-1}\right.$, in $\mathrm{CS}_{2}$ ) at the closest SD distance of $1.15 \mathrm{~m}$ and $\lambda=0.53 \mathrm{~nm}$ (Fig. 9). In the given conditions, using $3 \mathrm{~h}$ measuring time, the coherent scattering cross section of the sample could be measured reasonably well down to $0.002 \mathrm{~cm}^{-1}$. We point out here that the use of $\mathrm{CS}_{2}$ as solvent with its negligible incoherent scattering is only a particular case; measurements of the scattering of typical solutions of colloids, surfactants or polymers in deuterated solvents would present somewhat more of a challenge. The fitted radii of gyration $\left(R_{\mathrm{g}}\right)$ of $\mathrm{C}_{60}$ and $\mathrm{C}_{70}$ are $0.360 \pm 0.009$ and $0.404 \pm 0.006 \mathrm{~nm}$, respectively, which are very close to earlier reported values (Henderson,

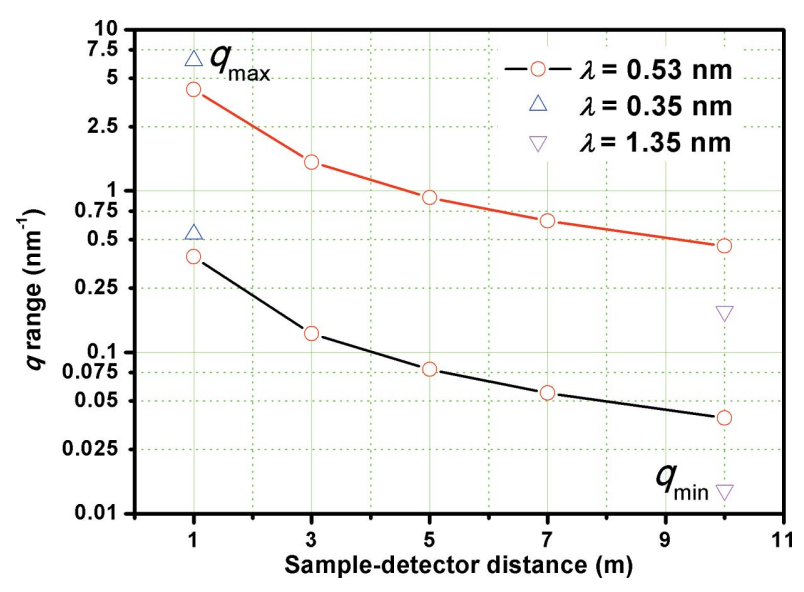

Figure 8

The accessible $q$ range calculated by $q=4 \pi \sin \theta / \lambda$ at different SD distances. Samples are typically measured at $\lambda=0.53 \mathrm{~nm}$ to get the maximum neutron flux.

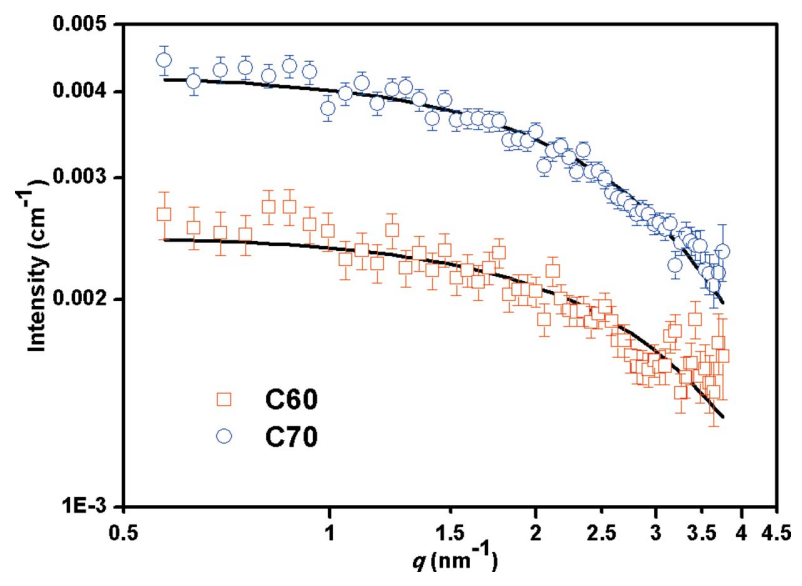

Figure 9

SANS scattering data obtained from $\mathrm{C} 60$ and $\mathrm{C} 70$ suspensions in $\mathrm{CS}_{2}$. Scattering of $\mathrm{CS}_{2}$ has been subtracted. The solid lines are the fits to the Guinier law. 
1997; Melnichenko et al., 1999; Migliardo et al., 2004, Avdeev et al., 2010).

A PMMA nanosphere suspension was used to determine the effective $q_{\min }$ of Suanni at the longest SD distance of $10.25 \mathrm{~m}$. As shown in Fig. 10(a), the lower limit of $q$ can reach $0.013 \mathrm{~nm}^{-1}$ at $\lambda=1.35 \mathrm{~nm}$ and $R_{2}=2 \mathrm{~mm}$, for samples with absolute scattering cross section of the order of $1000 \mathrm{~cm}^{-1}$. The data obtained from PMMA were fitted by a form factor of spheres with lognormal distribution of radius, and the instrumental smearing has been taken into account by convoluting the theoretical curve with the resolution function. The fitted median radius and standard deviation are $39.1 \mathrm{~nm}$ and 0.08 , respectively. In Fig. 10(b), the histogram was constructed by measuring radii of about 100 nanospheres in the scanning electron microscopy (SEM) image [inset to Fig. 10(a)]. The lognormal distribution fit to the histogram data gives a median radius and standard deviation of $38.5 \mathrm{~nm}$ and 0.05 , respectively. The SANS results are generally consistent with the data measured by SEM, though model fitting gives a broader particle size distribution. We suggest four possibilities for the observed differences: they could result from systematic errors and limited statistics of the SEM data; there may be systematic

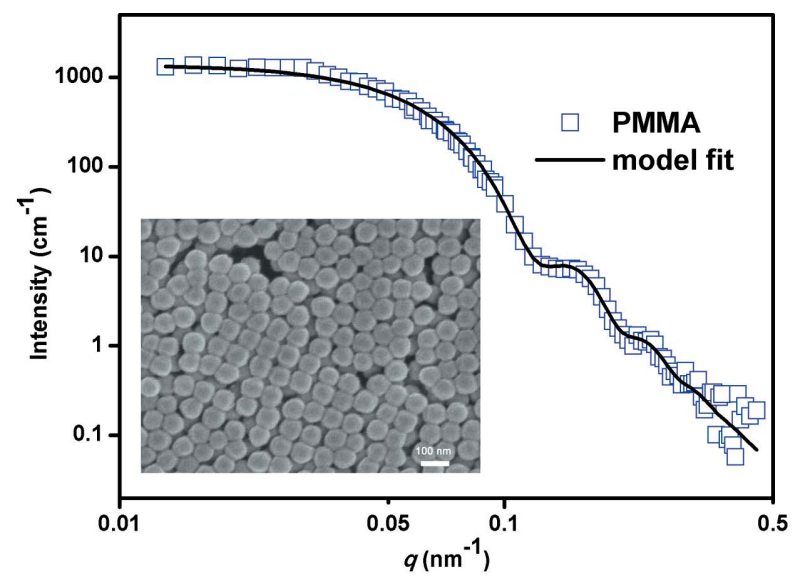

(a)

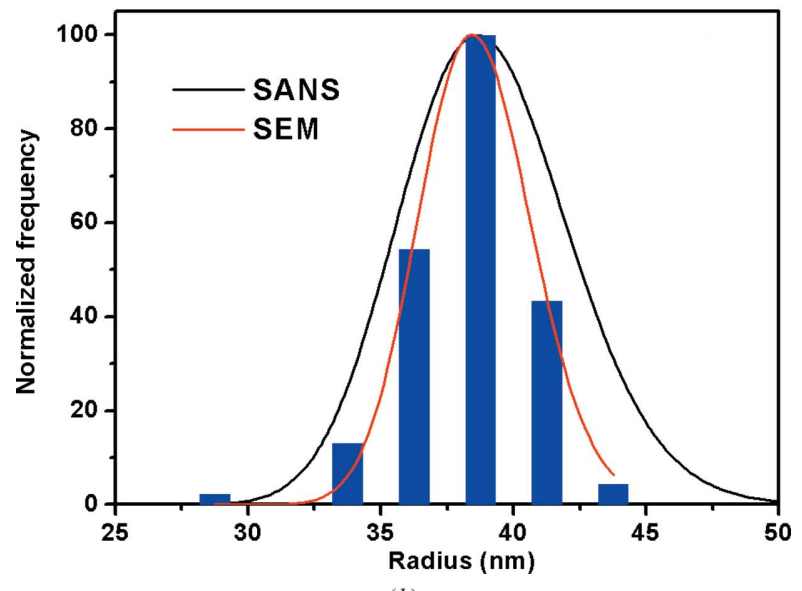

(b)

Figure 10

SANS scattering curve (a) and size distributions (b) of PMMA nanospheres. The inset shows a SEM image of PMMA. The histogram is the result of the statistical analysis of the SEM image. distortion caused by the higher sensitivity of scattering by the larger particles; the assumed lognormal distribution may be not fully correct, especially at the two ends of the size distribution; and finally the width of the resolution function could have been underestimated.

\section{Conclusions}

The wavelength, the scattering vector magnitude $q$ and the absolute intensity for the Suanni spectrometer have been calibrated by using standard samples. These measurements were validated by comparison with similar measurements on other instruments. The effective lower and upper $q$ limits of 0.013 and $5 \mathrm{~nm}^{-1}$ were determined by measurements of a PMMA suspension and a glassy carbon plate at the wavelengths of 1.35 and $0.35 \mathrm{~nm}$, respectively. A $g$ factor of 1.35 (to be used with $1 \mathrm{~mm}$-thick $\mathrm{H}_{2} \mathrm{O}$ at $\lambda=0.53 \mathrm{~nm}$ for absolute intensity scale calibration) has been determined by using an $\mathrm{h} /$ $\mathrm{d}$ polystyrene mixture. To increase the precision of the size parameters, $\lambda_{\mathrm{m}}$ should be used, especially for longer wavelengths $(\lambda>0.8 \mathrm{~nm})$. The suite of calibration methods and examples presented in this study provides information to enable precise data reduction and also to guide the design of experiments.

\section{Acknowledgements}

We thank the Paul Scherrer Institute (Switzerland), the Research Centre for Natural Sciences (Hungary) and the National Institute of Standards and Technology (USA) for providing beam time on SANS-2, CREDO and NG-7, respectively. We thank Professor Dong Qiu (from the Institute of Chemistry, Chinese Academy of Science) for providing the PMMA nanoparticle suspensions. We thank Mark J. Henderson for useful discussions and improving the English fluency of this communication.

\section{Funding information}

This work was supported by the National Natural Science Foundation of China under grant Nos. 11775195 (to QT), 11605171 (to DL) and 11705173 (to JC), the Scientific Research Fund of Southwest University of Science and Technology (18zx7108 to QT), the Director Foundation of the China Academy of Engineering Physics (YZ2015009 to CH), and the Science Challenge Project (JCKY2016212A504 to GY).

\section{References}

Allen, A. J., Zhang, F., Kline, R. J., Guthriec, W. F. \& Ilavsky, J. (2017). J. Appl. Cryst. 50, 462-474.

Almásy, L., Len, A., Markó, M. \& Rétfalvi, E. (2006). Z. Kristallogr. Suppl. 23, 211-216.

Avdeev, M. V., Tropin, T. V., Bodnarchuk, I. A., Yaradaikin, S. P., Rosta, L., Aksenov, V. L. \& Bulavin, L. A. (2010). J. Chem. Phys. 132, 164515.

Bates, F. T. \& Wignall, G. D. (1986). Phys. Rev. Lett. 57, 1429-1432. 
Breßler, I., Kohlbrecher, J. \& Thünemann, A. F. (2015). J. Appl. Cryst. 48, 1587-1598.

Brûlet, A., Lairez, D., Lapp, A. \& Cotton, J.-P. (2007). J. Appl. Cryst. 40, 165-177.

Dewhurst, C. D., Grillo, I., Honecker, D., Bonnaud, M., Jacques, M., Amrouni, C., Perillo-Marcone, A., Manzin, G. \& Cubitt, R. (2016). J. Appl. Cryst. 49, 1-14.

Gilles, R., Keiderling, U. \& Wiedenmann, A. (1998). J. Appl. Cryst. 31, 957-959.

Glinka, C. J., Barker, J. G., Hammouda, B., Krueger, S., Moyer, J. J. \& Orts, W. J. (1998). J. Appl. Cryst. 31, 430-445.

Hammouda, B. (2016). Probing Nanoscale Structures - The SANS Toolbox. National Institute of Standards and Technology, Center for Neutron Research, Gaithersburg, MD, USA.

Hammouda, B. \& Mildner, D. F. R. (2007). J. Appl. Cryst. 40, 250-259.

Henderson, S. J. (1997). Langmuir, 13, 6139-6145.

Karge, L., Gilles, R. \& Busch, S. (2017). J. Appl. Cryst. 50, 1382-1394.

Keiderling, U. (2002). Appl. Phys. A Mater. Sci. Process. 74, s1455s1457.

Keiderling, U., Gilles, R. \& Wiedenmann, A. (1999). J. Appl. Cryst. 32, 456-463.

Knoll, G. F. (2010). Radiation Detection and Measurement. New York: Wiley.

Lindner, P. (2000). J. Appl. Cryst. 33, 807-811.
Lindner, P. \& Zemb, Th. (2002). Neutron, X-rays and Light Scattering Methods Applied To Soft Condensed Matter. Amsterdam: NorthHolland.

Melnichenko, Y. B., Wignall, G. D., Compton, R. N. \& Bakale, G. (1999). J. Chem. Phys. 111, 4724-4728.

Migliardo, F., Magazù, V. \& Migliardo, M. (2004). J. Mol. Liq. 110, 3-6.

Nyam-Osor, M., Soloviov, D. V., Kovalev, Y. S., Zhigunov, A., Rogachev, A. V., Ivankov, O. I., Erhan, R. V. \& Kuklin, A. I. (2012). J. Phys. Conf. Ser. 351, 012024.

Peng, M. et al. (2016). Nucl. Instrum. Methods Phys. Res. A, 810, 6367.

Rosta, L. J., Füzi, J. \& Hományi, L. (2006). Physica B, 385-386, 1283 1286.

Schelten, J., Wignall, G. D., Ballard, D. G. H. \& Longman, G. W. (1977). Polymer, 18, 1111-1120.

Strunz, P., Mortensen, K. \& Janssen, S. (2004). Physica B, 350, E783E786.

Strunz, P., Šaroun, J., Keiderling, U., Wiedenmann, A. \& Przenioslo, R. (2000). J. Appl. Cryst. 33, 829-833.

Wacha, A., Varga, Z. \& Bóta, A. (2014). J. Appl. Cryst. 47, 1749-1754.

Wignall, G. D. \& Bates, F. S. (1987). J. Appl. Cryst. 20, 28-40.

Zhang, F., Ilavsky, J., Long, G. G., Quintana, J. P. G., Allen, A. J. \& Jemian, P. R. (2010). Metall. Mater. Trans. A, 41, 1151-1158. 